(c) 2015 IEEE. Personal use of this material is permitted. Permission from IEEE must be obtained for all other uses, in any current or future media, including reprinting/republishing this material for advertising or promotional purposes, creating new collective works, for resale or redistribution to servers or lists, or reuse of any copyrighted component of this work in other works.

DOI: 10.1109/MMS.2015.7375431 


\title{
A 77-GHz Cavity Antenna Array in PCB Technology
}

\author{
Claudia Vasanelli, Tobias Ruess, and Christian Waldschmidt \\ University of Ulm, Institute of Microwave Engineering, 89081 Ulm, Germany \\ Email: claudia.vasanelli@uni-ulm.de
}

\begin{abstract}
In this paper the design and the implementation of a cavity antenna array in PCB technology for automotive radar applications is presented. The array consists of four cavity antennas fed by a microstrip-line feeding network and $H$-shaped coupling apertures in the ground plane. Simulation and measurement results show that the proposed structure is a suitable candidate for the integration in automotive radar sensors.
\end{abstract}

\section{INTRODUCTION}

Driver assistance systems support the safety of the driver, help to avoid potentially car accidents, or try to mitigate their effects. A number of different sensors are employed like lidar, radar, or video [1]. Among them, radar sensors can measure accurately the radial velocity and distance of the surrounding vehicles, and they are robust against bad weather conditions.

In the last years, many different antenna concepts have already been proposed for the $76 \mathrm{GHz}-81 \mathrm{GHz}$ frequency band that is allocated in Europe for automotive radar systems. The main challenge about antenna array design for such applications is to achieve a sufficiently high gain, small dimensions, and a relative broad field of view, depending on the sensor application, by using standard fabrication technology suitable for mass production, i.e. printed circuit board (PCB) technology. On the other hand, it is a well-known problem that in the millimeter-wave frequency range planar circuits and antennas in PCB technology suffer of high losses and are sensitive to increased etching tolerances that could drastically reduce their performances. Thus, a wide bandwidth behavior is required to increase the robustness of the design.

Recently, promising cavity antenna arrays for radar applications have been presented [2], [3]. They show a wide impedance bandwidth, high gain, and a stable radiation pattern. Both configurations are designed on low temperature co-fired ceramic (LTCC), which is not suited for mass production of radar sensors. The LTCC technology shows indeed high inaccuracies in the fabrication process, like shrinking tolerances or possible layer misalignment [3], and moreover it shows usually high permittivity and relatively high losses. For these reasons, realizing efficient antenna arrays can be challenging [4].

This paper reports the implementation of a linear cavity antenna array employing standard PCB fabrication process and evaluates the feasibility of this antenna concept for automotive radar applications. To the best of the authors' knowledge, for the first time this kind of radiating element is developed on PCB technology.

\section{Antenna Array Design}

In Fig. 1 the layer stackup of the proposed structure is depicted. As it can be seen also from the 3D model in Fig. 2,

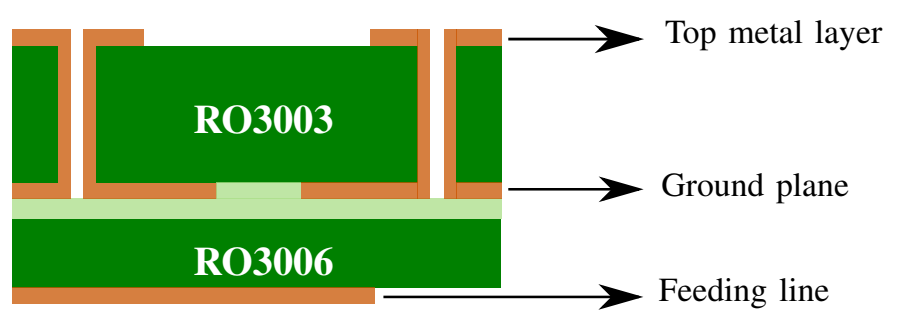

Fig. 1: Stackup of the proposed structure.

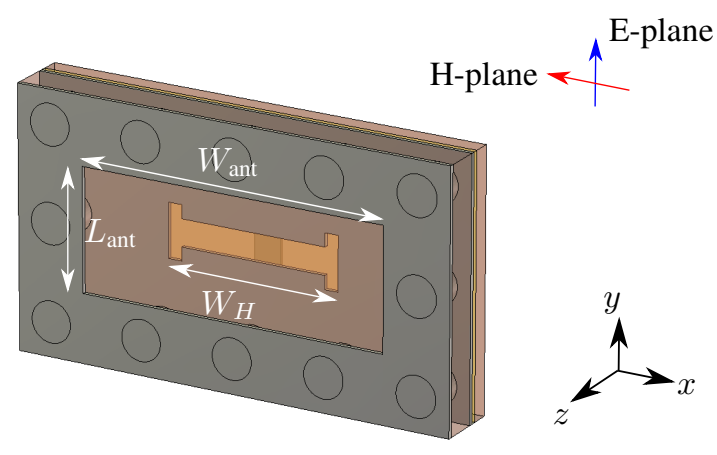

Fig. 2: 3D model of the single element. The H-shape slot in the ground plane is depicted in the center of the structure. The two main cuts of the radiation pattern are highlighted.

a two-layer structure is employed. The top layer is the cavity antenna surrounded by vias that are connecting the top metal layer and the ground plane. The cavity is designed to have the same properties as an open-ended integrated waveguide [2]. It has already been proved that waveguide-like structures can be realized by using via-fences in the substrate [5]. A $254 \mu \mathrm{m}-$ thick substrate (RO3003) from Rogers Corporation [6] and with permittivity $\varepsilon_{r}=3$ is employed. On the ground plane, an $\mathrm{H}$-shape slot allows the coupling from the feeding layer, i.e. from the microstrip line (MSL). To achieve a broad impedance matching, a resonant slot in the ground plane is designed. The length of the slot is $W_{H}=1.2 \mathrm{~mm}$. The drawback of this choice is the increased level of back radiation [7].

For reducing the spurious radiation from the feeding, a higher permittivity and thinner substrate is used (RO3006, $\varepsilon_{r}=6.15$, thickness $\left.127 \mu \mathrm{m}\right)$. Between the top and the feeding layer a bonding film is placed, namely RO3001. The dimensions of the cavity are $W_{\text {ant }}=1.9 \mathrm{~mm}=0.49 \lambda_{0}$ and $L_{\text {ant }}=0.7 \mathrm{~mm}=0.18 \lambda_{0}$, where $\lambda_{0}$ is the free-space wavelength at $77 \mathrm{GHz}$. Due to fabrication constraints, the diameter of the vias is $0.3 \mathrm{~mm}$ and the distance between the vias is also $0.3 \mathrm{~mm}$. 


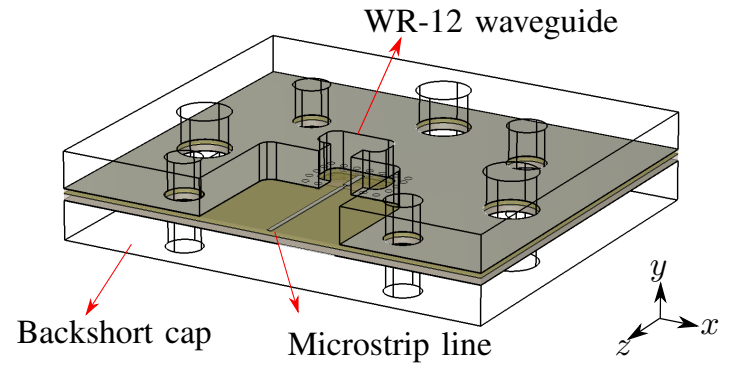

Fig. 3: 3D model of waveguide-to-microstrip line transition.

Starting from the aforementioned single element, a linear array of four elements has been designed. As already mentioned, the feeding network is realized in microstrip line technology. Simple Y-junction quarter-wave transformers are employed to split the input power to the four antenna elements. To avoid the presence of grating lobes, the distance between the single radiating elements is set to $2.9 \mathrm{~mm}$.

All the simulation results showed in this paper are obtained by means of a commercial 3D full-wave simulation software.

\section{A. Waveguide-to-Microstrip Line Transition}

To carry out the experimental characterization of the proposed structure, a proper transition from rectangular waveguide-to-microstrip line must be used and integrated with the antenna array. Many transitions have already been proposed in the literature. One of the most common examples is described in [8]. This kind of structure is simple, easy to fabricate, and it usually shows a broadband behaviour. However, it is mainly suited for single layer structure, while in the proposed structure a double-layer transition must be used.

Starting from the model proposed in [8], the waveguideto-microstrip line transition has been designed and optimized. The model of the transition is shown in Fig. 3. As can be seen, a microstrip probe is inserted inside the rectangular waveguide, which is connected perpendicularly to the PCB. On the opposite side, the transition is terminated by an external metal cap. The inner height of the metal cap is $80 \mu \mathrm{m}$. A number of grounded vias is placed around the microstrip probe.

In [8] it has been demonstrated that the probe can face either the waveguide or the backshort. To reduce the effect of the bulky waveguide on the radiation performance of the antenna array, in the proposed design the microstrip probe faces the waveguide.

The simulation results depicted in Fig. 4 shows that using a two-layer structure with different substrate parameters, in particular thicknesses and permittivities, has a great impact on the bandwidth and on the insertion loss of the transition. The achieved bandwidth is approximately $8 \mathrm{GHz}$ centered around $77 \mathrm{GHz}$. The maximum of the transmission coefficient amounts to $2.7 \mathrm{~dB}$.

\section{Measurement Results}

The fabricated antenna array can be seen in Fig. 5. The visual inspection of the manufactured prototypes showed roughly $20 \mu \mathrm{m}$ etching tolerances, that could of course affect

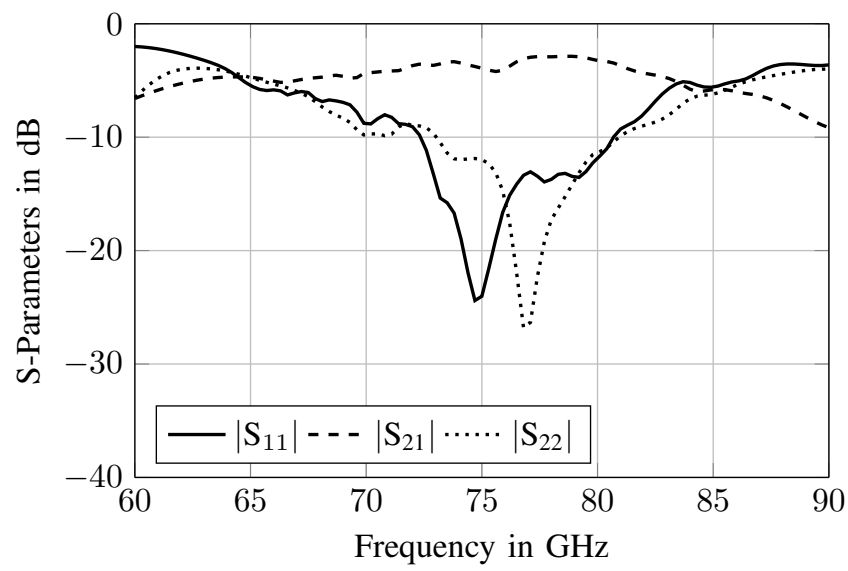

Fig. 4: Simulated scattering parameters of the waveguide-tomicrostrip line transition. Port 1 and 2 are placed at the end of the waveguide and of the microstrip line, respectively.

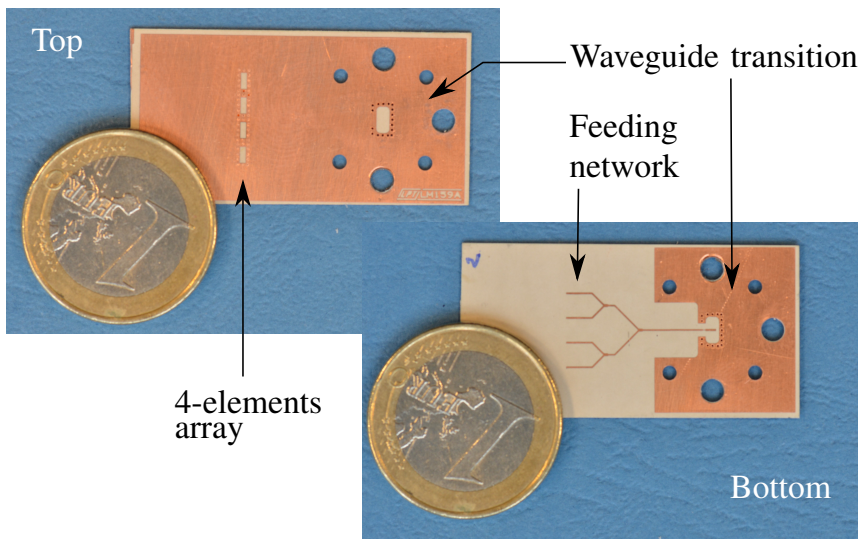

Fig. 5: Top and bottom view of the manufactured antenna.

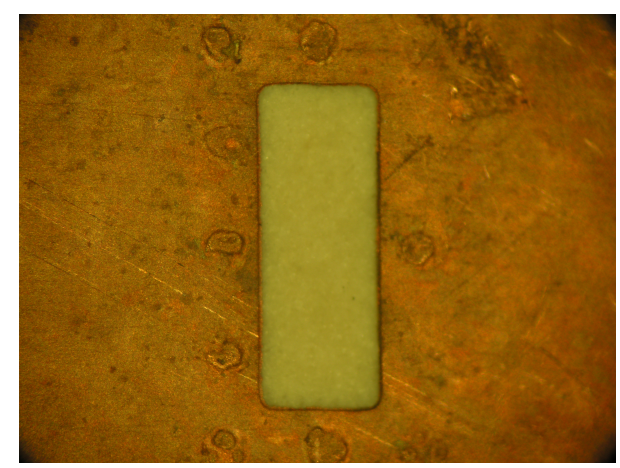

Fig. 6: Enlarged view of the single radiating element. 


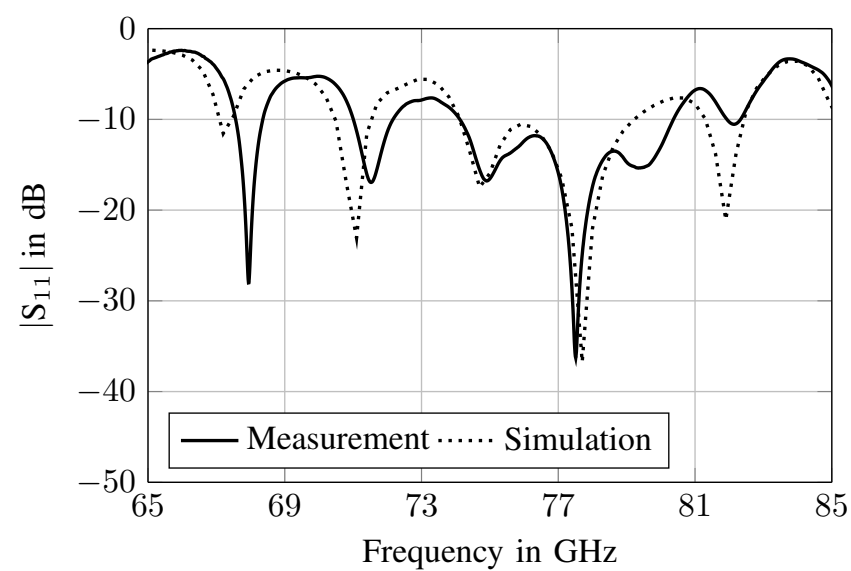

Fig. 7: Measured and simulated reflection coefficient of the antenna array integrated with the waveguide-to-microstrip transition.

the antenna impedance matching. A more detailed picture of the single radiating element can be seen in Fig. 6 .

The reflection coefficient and the radiation pattern of the array were then measured. In Fig. 7 it is depicted the comparison between the measured and the simulated reflection coefficient of the antenna array integrated with the waveguideto-microstrip line transition. The two curves are in good agreement and the measured bandwidth is approximately $6 \mathrm{GHz}$ centered around $77 \mathrm{GHz}$. It is worth mentioning that the substrates are characterized by the manufacturer only up to $10 \mathrm{GHz}$ and not in the millimeter-wave frequency range. Thus, it is most likely that the actual permittivities are different than the values reported in the data sheet [9]. Different simulations have been carried out to take into account possible different values of the relative permittivities. The simulation results reported in Fig. 7 refer to $\varepsilon_{r}=2.85$ for RO3003 and $\varepsilon_{r}=6$ for RO3006, instead of $\varepsilon_{r}=3$ and $\varepsilon_{r}=6.15$, respectively.

To remove the effect of the waveguide-to-microstrip line transition from the measured reflection coefficient, a time gating procedure has been applied. From Fig. 8 it can be observed that both a good agreement between measurements and simulations and a quite wide impedance matching in the desired frequency range are obtained.

Afterwards, the radiation pattern of the antenna array in the E- and H-plane has been measured. Figure 9 shows the fabricated antenna array placed in an anechoic chamber. During the measurement, the metal cap of the waveguide-tomicrostrip line transition has been coated by absorber material to reduce additional spurious reflections. Moreover, the frequency converter module has been covered by absorber material too, as it can be clearly seen from the picture.

The comparison of the measured and simulated radiation diagram in the H-plane is depicted in Figs. 10 and 11 for $77 \mathrm{GHz}$ and $79 \mathrm{GHz}$, respectively. A good agreement is obtained for both frequencies. The small shift of the measured main beam is due to a slight misalignment of the array on the turntable during the measurements.

The measured radiation pattern in the E-plane cut is broad

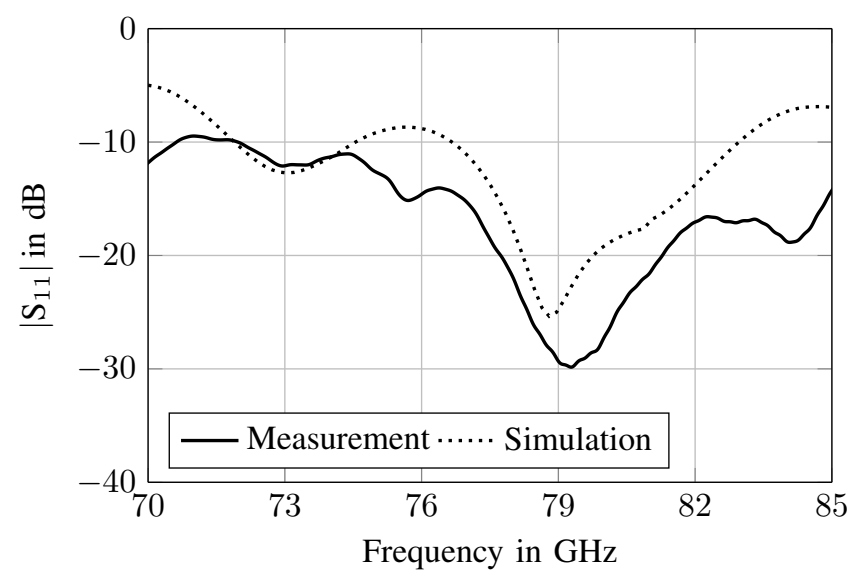

Fig. 8: Measured and simulated reflection coefficient of the antenna array. The effect of the transition has been removed by means of time gating.

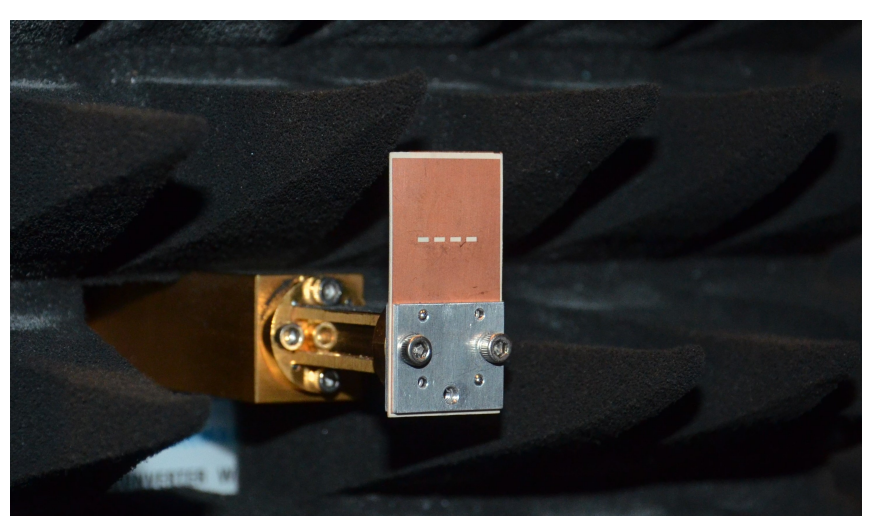

Fig. 9: Photo of the array placed in the anechoic chamber.

as expected, although the presence of the backshort metal cap has a slight effect during the measurements. Indeed, even if it has been coated with absorber material, it can still have an effect during the measurements. The main disadvantage of the proposed antenna array is the surface wave excitation caused by the behavior of the single radiating elements as an open-ended rectangular waveguide. A possible solution to solve this issue would be the design and the integration of an electromagnetic band gap structure to suppress surface waves effectively. Therefore, the outcomes of the surface wave excitation and of the presence of the metal cap may result in ripples in the farfield pattern.

By using a 25-dB standard horn antenna, the gain of the array has been measured and compared with the simulation results, as can be seen in Fig. 12. The differences in the measured and simulated values could be due to a wrong value of the loss tangent used in the numerical model. The measured gain at $77 \mathrm{GHz}$ is approximately $10 \mathrm{~dB}$. The simulated radiation efficiency is $67 \%$ at $77 \mathrm{GHz}$. 


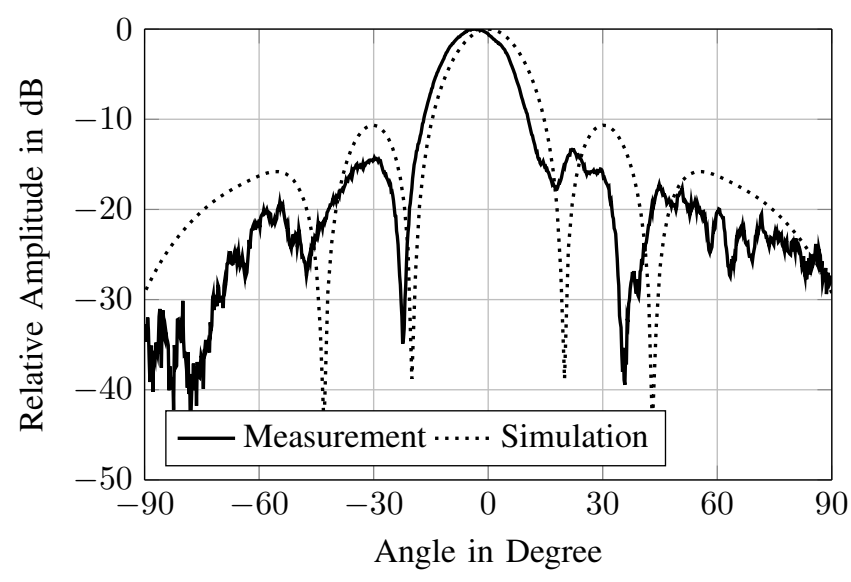

Fig. 10: Measured and simulated radiation diagram in the $\mathrm{H}$ plane at $77 \mathrm{GHz}$.

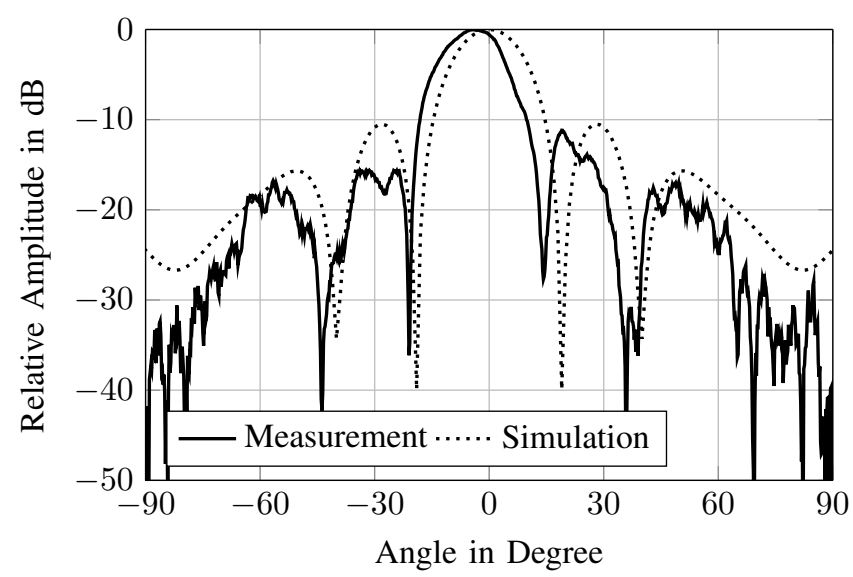

Fig. 11: Measured and simulated radiation diagram in the $\mathrm{H}$ plane at $79 \mathrm{GHz}$.

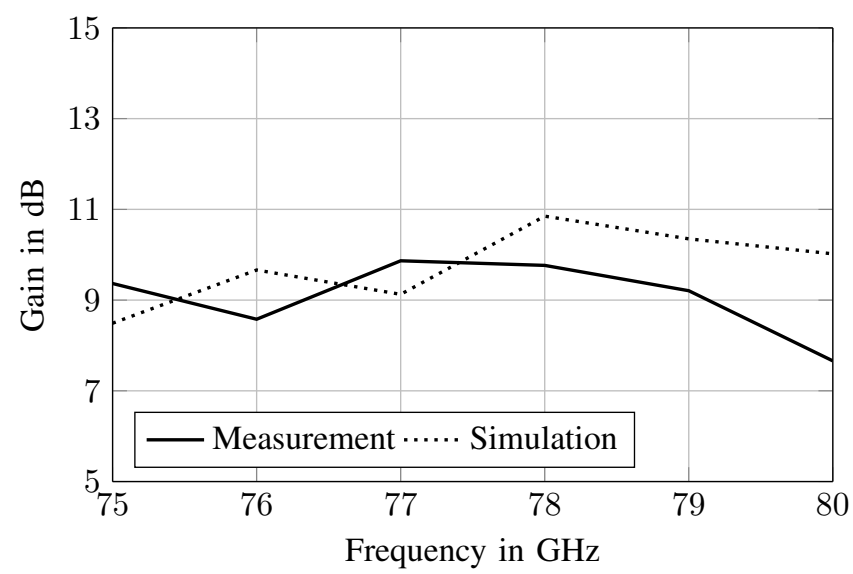

Fig. 12: Measured and simulated gain of the antenna array at boresight.

\section{CONCLUSiON}

In this paper, a four-element linear cavity antenna array fabricated in PCB technology has been presented for the first time. The simulation and measurement results of the reflection coefficient are in good agreement and prove that the array is able to cover the whole $77 \mathrm{GHz}$ frequency band for automotive radar sensors. The measured radiation pattern is also in good agreement with the simulations, although the surface wave excitation could affect the smoothness of the pattern in the E-plane.

\section{REFERENCES}

[1] J. Hasch, E. Topak, R. Schnabel, T. Zwick, R. Weigel, and C. Waldschmidt, A Millimeter-Wave Technology for Automotive Radar Sensors in the $77 \mathrm{GHz}$ Frequency Band, IEEE Trans. Microwave Theory Tech., vol. 60, no. 3, pp. 845-860, 2012.

[2] F. Bauer and W. Menzel, A 79-GHz Planar Antenna Array Using Ceramic-Filled Cavity Resonators in LTCC, IEEE Antennas Wireless Propagat. Lett., vol. 12, pp. 910-913, 2013.

[3] S. Beer, L. Pires, C. Rusch, C. Heine, T. Zwick, and J. Paaso, Microstrip slot antenna array in LTCC technology for a $122 \mathrm{GHz}$ systemin-package, in IEEE Antennas and Propagation Society International Symposium (APSURSI), pp. 1-2, 2012.

[4] F. Bauer, X. Wang, W. Menzel, and A. Stelzer, A 79-GHz Radar Sensor in LTCC Technology Using Grid Array Antennas, IEEE Trans. Microwave Theory Tech., vol. 61, no. 6, pp. 2514-2521, 2013.

[5] B. Sanadgol, S. Holzwarth, A. Milano, R. Popovich, $60 \mathrm{GHz}$ substrate integrated waveguide fed steerable LTCC antenna array, in Proc. of the Fourth European Conference on Antennas and Propagation (EuCAP), pp. 1-4, 2010.

[6] Rogers Corporation, CT 06263, USA.

[7] S. D. Targonski, R. B. Waterhouse, and D. M. Pozar. Design of wideband aperture-stacked patch microstrip antennas, IEEE Trans. Antennas Propagat., vol. 46, no. 9, pp. 1245-1251, 1998.

[8] J. Machac and W. Menzel. On the design of waveguide-to-microstrip and waveguide-to-coplanar line transitions, in Proc. of the 23rd European Microwave Conf., pp. 615-616, 1993.

[9] E. Topak, J. Hasch, and T. Zwick, Compact Topside Millimeter-Wave Waveguide-to-Microstrip Transitions, IEEE Microwave Wireless Compon. Lett., vol. 23, no. 12, pp. 641-643, 2013. 